\title{
Aplicação do conceito de Smart Cities na Cidade de Recife-PE
}

\author{
Application of the Smart Cities concept in the City of Recife-PE \\ Aplicación del concepto de Ciudades Inteligentes en la Ciudad de Recife-PE
}

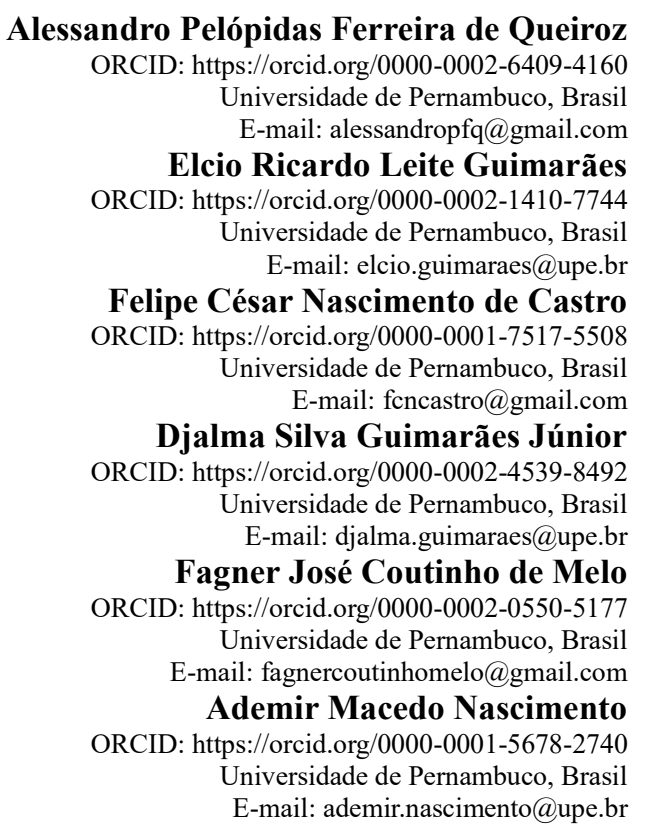

\section{Resumo}

Os avanços tecnológicos passam a fazer parte das rotinas das cidades, que possibilitam a oferta de serviços com foco em melhorias para a vida da população que ali habita. Para que determinada cidade seja reconhecida como Cidade Inteligente, faz-se indispensável o preenchimento de requisitos específicos denominados de indicadores. Para tanto, o presente artigo tem como objetivo identificar evidências da aplicação de conceitos de cidades inteligentes no Recife. Quanto à metodologia, trata-se de uma abordagem qualitativa, do tipo exploratória, onde a técnica de coleta de dados será a revisão de literatura, a partir de fontes secundárias, utilizando-se da análise de conteúdo para estudar as informações obtidas. Nesse sentido, verifica-se que o Índice de Desenvolvimento Humano Municipal - IDHM é uma ferramenta bastante utilizada para estimular a mensuração, a discussão e a conceituação de "desenvolvimento" da forma mais ampla, por intermédio de indicadores socioeconômicos nacionais, considerando temas como saúde, educação, renda, trabalho, habitação e vulnerabilidade. Diante disso, usando o IDHM como referência, é possível afirmar que o Estado de Pernambuco ocupa a $17^{\mathrm{a}}$ posição no ranking nacional, e por sua vez, Recife ocupa a $53^{\mathrm{a}}$ posição perante todos os municípios Pernambucanos, com o IDHM de 0,772.

Palavras-chave: Smart Cities; Indicadores; Tecnologia; Recife.

\begin{abstract}
Technological advances are now part of the routines of cities, which make it possible to offer services focused on improving the lives of the population that lives there. For a given city to be recognized as a Smart City, it is essential to fulfill specific requirements called indicators. Therefore, this article aims to identify evidence of the application of smart city concepts in Recife. As for the methodology, it is a qualitative approach, of the exploratory type, where the data collection technique will be the literature review, from secondary sources, using content analysis to study the information obtained. In this sense, it appears that the Municipal Human Development Index - IDHM is a widely used tool to stimulate the measurement, discussion, and conceptualization of "development" in the broadest way, through national socioeconomic indicators, considering topics such as health, education, income, work, housing and vulnerability. Therefore, using the IDHM as a reference, it is possible to affirm that the State of Pernambuco occupies the 17th position in the national ranking, and in turn, Recife occupies the 53rd position among all Pernambuco municipalities, with the IDHM of 0.772 .
\end{abstract}

Keywords: Smart Cities; Indicators; Technology; Recife. 


\begin{abstract}
Resumen
Los avances tecnológicos son ahora parte de las rutinas de las ciudades, que permiten ofrecer servicios enfocados a mejorar la vida de la población que allí vive. Para que una determinada ciudad sea reconocida como Smart City, es fundamental cumplir unos requisitos específicos denominados indicadores. Por lo tanto, este artículo tiene como objetivo identificar evidencia de la aplicación de conceptos de ciudad inteligente en Recife. En cuanto a la metodología, se trata de un abordaje cualitativo, de tipo exploratorio, donde la técnica de recolección de datos será la revisión de la literatura, de fuentes secundarias, utilizando el análisis de contenido para estudiar la información obtenida. En este sentido, parece que el Índice de Desarrollo Humano Municipal - IDHM es una herramienta ampliamente utilizada para estimular la medición, discusión y conceptualización del "desarrollo" de la manera más amplia, a través de indicadores socioeconómicos nacionales, considerando temas como salud, educación, ingresos, trabajo, vivienda y vulnerabilidad. Por lo tanto, tomando como referencia el IDHM, es posible afirmar que el Estado de Pernambuco ocupa el puesto 17 en el ranking nacional y, a su vez, Recife ocupa el puesto 53 entre todos los municipios de Pernambuco, con el IDHM de 0,772 .
\end{abstract}

Palabras clave: Ciudades inteligentes; Indicadores; Tecnología; Recife.

\title{
1. Introdução
}

As cidades passaram a implementar os avanços tecnológicos para oferecer produtos e serviços de modo a melhorar a qualidade de vida dos seus habitantes (Damiani et al. 2019). Em decorrência da apropriação da tecnologia com tal finalidade estas cidades passaram a ser identificadas como cidades inteligentes (Bachendorf, 2018). E de acordo com Lemos (2013, p. 48), inteligente "é sinônimo de uma cidade na qual tudo é sensível ao ambiente e produz, consome e distribui um grande número de informações em tempo real."

Segundo o IBGE (2020) estima-se a população brasileira é de aproximadamente 213 milhões, e que a maior parte, ou seja, 84,72\%, vive em áreas urbana (IBGE, 2015), é compreensível que as lideranças comecem a pensar, elaborar e implementar, através das tecnologias disponíveis, estratégias para proporcionar melhorias aos habitantes, especialmente em cidades que já dão sinais de que necessitam desse tipo de investimento.

No estado de Pernambuco, a população estimada é de aproximadamente 9,5 milhões de habitantes (IBGE, 2020). Para se ter uma noção, a Região Metropolitana (RM) de Recife, institucionalizada pela Lei Federal n. 14 de 1973, é formada por 14 municípios: Abreu e Lima, Araçoiaba, Camaragibe, Cabo de Santo Agostinho, Goiana, Igarassu, Ilha de Itamaracá, Ipojuca, Itapissuma, Jaboatão dos Guararapes, Moreno, Olinda, Paulista, Recife, São Lourenço da Mata. De acordo com IBGE (2010) na RM de Recife se concentram 3.589.674 habitantes residentes da zona urbana, ou seja, em 2010 compreendia a $51 \%$ da população urbana de Pernambuco.

Ademais, oportuno considerar, que quanto se refere a aumento da população, com ela surgem outras consequências, como por exemplo, aumento de veículos nas ruas das cidades. No período de 2010 a 2019 a poluição do Recife cresceu 7,02\% totalizando 1.645.727 habitantes, no entanto a frota cresceu para 687,793 veículos, ou seja, a proporção de veículos por habitante era de 0,32 em 2010 e em 2019 passou a ser de 0,42. (IBGE, 2018).

O crescente aumento da população e de veículos, implica na necessidade de criação de novos serviços, de médio e longo prazos, para garantir a mobilidade sustentável, a partir de iniciativas locais, preocupação que se insere no conceito das cidades identificadas como Smart City (Cidade Inteligente). Ainda, não se deve esquecer que, a mobilidade está inter-relacionada com outros setores, tais como o planejamento urbano, a saúde e a preservação ambiental, o que possibilita diálogos que envolvam tecnologia e inovação social, também presentes nas cidades inteligentes.

O El País, em 2018, publicou matéria com o seguinte título: Recife, o pior trânsito "em linha reta" (ROSSI, 2018). Para os recifenses, a notícia não os surpreendeu. A título de ilustração, nos períodos das $7 \mathrm{~h}$ às $10 \mathrm{~h}$ e das $17 \mathrm{~h}$ às $20 \mathrm{~h}$, deslocamentos de veículos na Capital pernambucana demoram em média 86\% mais tempo a serem realizados em comparação com períodos sem congestionamento (Toledo, 2017). Já em 2012, a média anual dos congestionamentos nos dias úteis, em km, nos horários de pico, chegava a 18,85 km/h (REDE, 2012). 
Nesse sentido, observa-se que a mobilidade urbana é uma das situações que precisam, com urgência, da iniciativa da Prefeitura para propositura de soluções para o problema. Contudo, além do planejamento urbano, outras tantas situações, como planejamento social e da administração pública também permanecem sem solução, considerando a extensão do conceito de cidades inteligentes. Para tanto, o presente artigo tem como objetivo identificar evidências da aplicação de conceitos de cidades inteligentes na cidade de Recife e analisar a sua relação com o desempenho do Índice de Desenvolvimento Humano Municipal (IDHM).

\section{Revisão da Literatura}

Portanto, na revisão da literatura o artigo será dividido em duas seções secundárias. Na primeira será realizada uma abordagem a respeito do conceito ou conceitos de uma Smart City. Na segunda, serão apresentados os indicadores usualmente utilizados para classificar as cidades como Smart City. Desse modo, a partir do embasamento teórico será possível realizar a análise qualitativa dos indicadores aqui escolhidos e verificar se há um conceito comum que possa ser analogamente adaptado e aplicado às cidades, tendo a cidade do Recife como um dos parâmetros para esta análise.

\subsection{As Smart Cities (Cidades Inteligentes)}

Com o crescimento desordenado das grandes aglomerações urbanas, o cotidiano das pessoas que vivem nas metrópoles vem passando por severas transformações em um curto espaço de tempo (Santos, 2000). Trânsito caótico e longos deslocamentos entre residência e trabalho é um dos principais desafios que se deve enfrentar de maneira imediata por, praticamente todas as grandes cidades do mundo (Harvey, 1992). O desenvolvimento de Smart City (Cidade Inteligente), pode ser uma das soluções para minimizar a problemática urbana nessas cidades.

Segundo Fernanda Rizzon, (2017) em seu artigo Smart City: um conceito em construção, a expressão foi popularizada na década de 90, quando tentava elaborar um conceito para indicação de uma Cidade Inteligente, levou a autora a construir um pensamento que convergisse ao pensamento desenvolvimentista, com aplicação e uso de tecnologias de informação e comunicação (TIC).

Em virtude da estreita ligação com a tecnologia, diversos indicadores surgiram para caracterizar as Cidades Inteligentes, criando espaço no desenvolvimento do conceito e dificultando a caracterização dos termos propostos. Como as questões de desenvolvimento, acesso a tecnologia e qualidade de vida. Questões não apenas locais, mas também são questões globais que perpassam o planejamento urbano e os grandes aportes financeiros é necessário para definir os diversos indicadores e especificidades de cada Cidade Inteligente. Smart City é um conceito em construção. Como forma de tornar os serviços da cidade e seu monitoramento mais eficazes, interativos e eficientes (Rizzon, 2017).

Os resultados apontados pela autora mostraram que as Cidades Inteligentes precisam distribuir tecnologias de informação e comunicação de forma compartilhada, não só em termos de recursos técnicos, em busca de qualidade de vida. (Rizzon, 2017).

A construção de Cidades Inteligentes visa integrar, de maneira sustentável, elementos como: mobilidade, tecnologia, saúde, educação e qualidade de vida (Harrison; Donnelly, 2011; Washburn, 2010). A maioria das definições menciona a necessidade de usar a tecnologia da informação para otimizar a infraestrutura urbana, gestão de recursos e uso de serviços urbanos (Harrison; Donnelly, 2011; Washburn, 2010). Algumas dessas definições ainda aumentam as necessidades de desenvolvimento sustentável da cidade e melhoram o uso de recursos como água e eletricidade (Caragliu 2011; Dameri, 2017).

Um dos aspectos relacionados, é que as cidades inteligentes também precisam promover o crescimento econômico urbano (Dameri, 2017) para integrar toda a população na sociedade. Duas definições se referem ao processo pelo qual a sociedade participa da tomada de decisão governamental por meio do governo participativo (Dameri, 2017; Giffinger; Haindlmaier, 2010). 
Outras questões importantes levantadas por esta definição são o monitoramento da infraestrutura urbana, como ruas, pontes, linhas de trem (Hall, 2000), o monitoramento da utilização de recursos como água e eletricidade (Hall, 2000), e a seleção de todos os serviços na cidade. Integração (Harrison; Donnelly, 2011; Washburn, 2010).

O Estudo da Urban Systems em 2020 aponta o ranqueamento de Cidades Inteligentes para algumas metrópoles brasileiras, com base em um conjunto de indicadores. No estudo em questão o objetivo é definir as cidades com maior potencial de desenvolvimento do Brasil. O resultado, publicado pela Connected Smart Cities e Mobility Digital Xperience 2020, traz indicadores baseados na ISO 37122 - Sustainable Cities And Communities - Indicators For Smart Cities. O resultado é apresentado em quatro frentes: geral, por eixo temático, por região e por faixa populacional. O estudo é composto por indicadores de 11 principais setores: mobilidade, urbanismo, meio ambiente, tecnologia e inovação, economia, educação, saúde, segurança, empreendedorismo, governança e energia. Com base nesse conjunto de indicadores, a $1^{a}$ colocação no Ranking Smart Cities no Nordeste é a cidade do Recife - PE

Quadro 1 - Posição Município - UF/Nota.

\begin{tabular}{|c|c|c|}
\hline $1^{\circ}$ & São Paulo - SP & 37,901 \\
\hline $2^{\circ}$ & Florianópolis - SC & 37,224 \\
\hline $3^{\circ}$ & Curitiba - PR & 36,545 \\
\hline $4^{\circ}$ & Campinas - SP & 36,303 \\
\hline $5^{\circ}$ & Vitória - ES & 36,251 \\
\hline $6^{\circ}$ & São Caetano do Sul - SP & 36,107 \\
\hline $7^{\circ}$ & Santos - SP & 35,423 \\
\hline $8^{\circ}$ & Brasília - DF & 35,361 \\
\hline $9^{\circ}$ & Porto Alegre - RS & 34,869 \\
\hline $10^{\circ}$ & Belo Horizonte - MG & 34,608 \\
\hline $11^{\circ}$ & Niterói - RJ & 34,411 \\
\hline $12^{\circ}$ & Rio de Janeiro - RJ & 34,297 \\
\hline $13^{\circ}$ & Barueri - SP & 34,214 \\
\hline $14^{\circ}$ & Campo Grande - MS & 34,002 \\
\hline $15^{\circ}$ & Recife - PE & 33,557 \\
\hline $16^{\circ}$ & Balneário Camboriú - SC & 33,449 \\
\hline $17^{\circ}$ & Jaguariúna - SP & 33,421 \\
\hline $18^{\circ}$ & Itajaí - SC & 33,078 \\
\hline $19^{\circ}$ & Blumenau - SC & 33,017 \\
\hline $20^{\circ}$ & São José dos Campos - SP & 32,979 \\
\hline
\end{tabular}

Fonte: Urban Systems (2020).

\subsection{O IDH e IDHM como Indicador de Cidade Inteligente}

Um índice pode ser considerado como indicador aproximado para Cidades Inteligentes é o Índice de Desenvolvimento Humano (IDH) e o Índice de Desenvolvimento Humano Municipal (IDHM) criados pelo Programa das Nações Unidas para o Desenvolvimento (PNUD) são índices baseados em medidas econômicas, culturais, políticas e sociais e são usados para quantificar o desenvolvimento de uma determinada população. Portanto, para explicar o que é IDHM, faz-se necessária uma breve introdução do IDH.

O IDH foi publicado pela primeira vez em 1990, seus cálculos são baseados no tripé PIB per capita (indicador de renda), expectativa de vida (indicador de saúde) e taxa de analfabetismo (indicador de educação). Esses números representam os dois anos anteriores ao lançamento da análise. Portanto, o IDH de 1988 retrata a cena de 1990. Nesse sentido, Torres (2016) destacou que a construção desse novo indicador de desenvolvimento reflete uma estreita relação com o debate em torno da relação do homem e sua relação com a cidade onde ele vive, o que irá afetar diretamente na sua qualidade de vida e nos indicadores sobre 
essa temática. Partem do reconhecimento de que a qualidade de vida não se limita ao campo econômico para se indicar que uma cidade é inteligente ou não (Torres, 2016).

Em 2010, 20 anos depois, o IDH passou por algumas mudanças. Exemplo, o Produto Interno Bruto (PIB) per capita, por exemplo, foi substituído pela Renda Nacional Bruta (RNB) per capita, a qual contabiliza a renda conquistada pelos residentes de um país, incluindo fluxos internacionais, como remessas vindas do exterior e ajuda internacional, e excluindo a renda gerada no país, mas repatriada ao exterior. Ou seja, a RNB apresenta um retrato mais preciso do bem-estar econômico da população de um país (PNUD, 2010).

Da mesma forma, o índice de educação foi revisado para substituir a taxa de analfabetismo pelo cálculo da média de anos de educação da população adulta ( 25 anos ou mais). Outra mudança importante é que os dados fornecidos pelo novo método envolvem previsões feitas para o ano corrente. Portanto, o Índice de Desenvolvimento Humano de 2010 é calculado com base nas projeções para o mesmo ano. (Relatório de Desenvolvimento Humano de 2010).

Com base na metodologia do IDH, o IDHM visa medir a escala de desenvolvimento econômico e social em menor escopo, na municipalidade. (PNUD, 2013).

Segundo o estudo do PNUD em 2013, diferentemente do IDH, para o IDHM, foram estabelecidas cinco faixas de desenvolvimento ao invés de quatro, a saber:

- Muito baixo - entre 0,0 e 0,499;

- Baixo - entre 0,500 e 0,599;

- $\quad$ Médio - entre 0,600 e 0,699;

- Alto - entre 0,700 e 0,799 ; e

- $\quad$ Muito alto - entre 0,8000 e 1,0 .

A escala apresentada acima, encontra-se diretamente ligada à maturidade de um município, sendo um dos principais indicadores para apontar uma cidade como inteligente (PNUD, 2013).

É o IDHM que pode determinar a existência e a extensão de desigualdades existentes na população e facilitar políticas públicas para solucionar tal problemática. Em grandes áreas, a probabilidade de se criar situação de apartheid é alta, portanto, a aplicação do IDHM deve ser em menor escala e para poder identificar situação com mais clareza, além disso, a necessidade de novos indicadores é fundamental para fazer parte de políticas públicas e tornar os processos de tomada de decisão cada vez mais racionais (Torres, 2016). Nesse sentido o IDHM se torna um dos indicadores fundamentais para delinear questões sociais nacionais ou locais no tocante a Cidades inteligentes (Torres, 2016).

A expansão do gasto público está relacionada ao seu papel na produção de bens públicos e ao controle das externalidades em uma economia de mercado, pois, conforme explica Rezende (2000), "dado que o mercado não pode se organizar efetivamente para produzir bens públicos, o governo tende a se expandir. "É papel do estado garantir o estado de bem-estar social, condição fundamental para se perseguir o caminho de uma Cidade Inteligente (Torres, 2016).

Basicamente, segundo Slomski (2003) a regulação é a busca do melhor em qualquer entidade (seja ela pública ou privada). É perseguido por um conjunto de elementos que constituem um norte de qualquer entidade, Slomski (2003). Nesse norte, encontra-se as boas práticas na busca por cidades inteligentes. A tarefa desta regulamentação é difundir conhecimento, construir modelos e implementar sistemas de informação que possam transformar cidades comuns em Cidades Inteligentes, além de responder aos usuários com informações econômicas, físicas e financeiras. Este regulamento é responsável por garantir a perfeita execução dos processos de tomada de decisão, ação, informação e controle, acompanhamento e controle das atividades das cidades inteligentes (Torres, 2016). 
Para aplicar o IDHM na busca por uma Cidade Inteligente, os gestores eleitos da população têm a responsabilidade de gerir as finanças públicas para que as despesas se desenvolvam ao máximo, porque as despesas públicas, essas estão relacionadas com o desenvolvimento e qualidade de vida. (Torres, 2016).

Portanto, é dever dos gestores públicos zelar pelos gastos públicos. Haja vista que gastos e receitas públicas afetam o IDHM e consequentemente a busca por uma Cidade Inteligente. Nesse norte, é fundamental a instituição de órgãos de controle municipal, para que através do monitoramento das finanças públicas, possam garantir a maior efetividade dos implementos de políticas públicas. (Torres, 2016).

\section{Metodologia}

A metodologia utilizada nesta pesquisa é a abordagem qualitativa, a qual possibilita a análise e interpretação por parte do pesquisador, com suas apreciações sobre o fenômeno em estudo (Pereira et al., 2018). O estudo também se valerá da pesquisa exploratória, com o objetivo de investigar um problema, para fornecer informações mais precisas, bem como, permitir maior proximidade com o tema (Gil, 2007).

A pergunta norteadora foi identificar oportunidades de iniciativas de cidades inteligentes em Recife, considerando as perspectivas tecnológicas, do planejamento urbano, social e da administração pública.

Nesse sentido, para elaboração do presente estudo, foi utilizada uma revisão de literatura do tipo narrativa abrangendo livros, artigos científicos, sites e notícias, monografias, dissertações e teses publicadas e disponíveis nas bases de dados: Periódicos Capes (Coordenação de Aperfeiçoamento de Pessoal de Nível Superior), Scielo (Scientific Eletronic Library) e Google Acadêmico. Vale destacar que os estudos que não se enquadravam na temática da pesquisa foram descartados.

Para a realização da busca, utilizou-se os seguintes descritores: "Cidades Inteligentes", "Smart Cities", "Smart City" e "Indicadores". Ademais, esclarece-se a necessidade de uso do operador booleano AND para conectar as palavras aqui descritas.

Ainda que, de acordo com Rother (2007) a revisão narrativa de literatura não exija que informações correspondentes às fontes utilizadas, a metodologia para a busca das referências, e nem os critérios utilizados na avaliação e seleção dos trabalhos, para a pesquisa em questão, foram seguidas as etapas aqui relacionadas: definição do tema e elaboração do problema de pesquisa; elaboração dos critérios de elegibilidade, inclusão e exclusão dos estudos; levantamento das publicações nas bases de dados; categorização e análise das informações encontradas nas publicações; avaliação dos estudos selecionados; apresentação dos resultados, incluindo análise crítica dos achados e síntese da revisão (Silva et al. 2020).

A presente pesquisa assegura os aspectos éticos, comprometendo-se com a autoria dos materiais pesquisados, estando os autores citados tanto no presente artigo como nas respectivas referências ao final.

\section{Resultados e Discussão}

Diante de tantas metodologias propostas para "classificar as cidades como Smart Cities - Cidades Inteligentes", entendese que há uma tentativa importante de se obter uma metodologia que possa servir de referência e com base de aplicação viável para ser comparada em qualquer local, onde haja indicadores que possibilitem a análise de dados e se obter resultados.

Portanto, este artigo pretende identificar estudo que permita essa comparabilidade com base em informações, cujo padrão seja aceito e reconhecido pelo setor público, privado, instituições, mundo acadêmico, estudiosos e pessoas interessadas na análise comparativa entre locais, cidades, regiões e países.

Nessa direção, destaca alguns trabalhos que classificam as cidades como inteligentes e percebe que o sentido final de se estabelecer a análise sobre as cidades serem ou não inteligentes, está diretamente ligada a aspectos de desenvolvimento educacional, urbano, social, econômico e de governança. Então, para desenvolver a proposta aqui relatada, foi realizado um levantamento qualitativo de informações sobre cidades inteligentes, nas fontes citadas no item de referências. Essas informações 
foram coletadas estritamente para o objetivo deste artigo, sendo, portanto, limitadas ao âmbito deste trabalho, para dar subsídio às indagações e propostas que se pretende. Em função disso, detalha a partir deste capítulo o IDHM, um estudo que já foi iniciado a mais de uma década, denominado a seguir.

O Atlas do Desenvolvimento Humano no Brasil 2013 e o cálculo do IDHM é uma ferramenta já consagrada para estimular a mensuração, a discussão e a conceituação de "desenvolvimento" da forma mais ampla (Chediek et al. 2013).

O Atlas 2013 aponta caminhos a seguir no futuro, ao narrar de forma sintética as transformações sociais alcançadas local, regional e nacionalmente entre os censos realizados pelo Instituto Brasileiro de Geografia e Estatística - IBGE em 1991, 2000 e 2010. Os indicadores facilitam o diálogo objetivo entre usuários de toda parte, sobre diferenças e semelhanças nas nossas jornadas humanas em busca de progresso (Chediek et al. 2013).

O novo Atlas Brasil 2013 tenta trazer de forma simples e clara, o Índice de Desenvolvimento Humano Municipal dos municípios brasileiros, e ainda mais de duas centenas de indicadores socioeconômicos nacionais, percorrendo temas como saúde, educação, renda, trabalho, habitação e vulnerabilidade (Chediek et al. 2013).

O IDHM é calculado em função de três pilares fundamentais: vida longa e saudável, acesso ao conhecimento e qualidade de vida (Chediek et al. 2013). Por vida longa e saudável se entende a formulação do primeiro índice IDHM LONGEVIDADE (1) - expectativa de vida ao nascer. Por acesso ao conhecimento se forma o segundo índice IDHM EDUCAÇÃO (2) com as variáveis - Escolaridade da população adulta e fluxo escolar da população jovem. O terceiro indicador, denominado índice IDHM RENDA (3) - padrão de vida, constitui a terceira variável que é a renda per capita. Daí se extrai a primeira fórmula que é a raiz cúbica da multiplicação dos subíndices com pesos um e dois, que formam o índice IDHM EDUCAÇÃO de acesso ao conhecimento, obtendo-se assim o resultado da raiz cúbica que é a média geométrica dos seus dois subíndices:

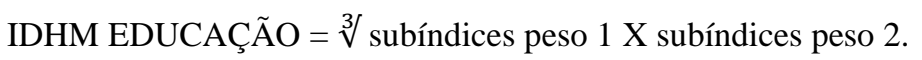

Dessa forma o Índice de Desenvolvimento Humano Municipal - IDHM é dado pelo resultado da raiz cúbica dos três índices citados acima:

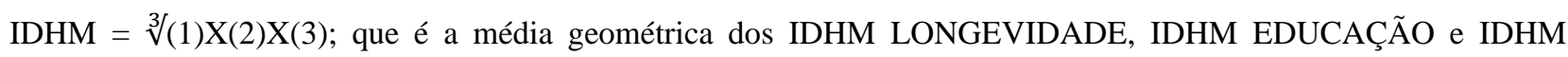
RENDA.

O IDHM LONGEVIDADE (1) que é extraído da expectativa de vida ao nascer, é calculada por método indireto, através dos dados dos Censos Demográficos do IBGE. Esse indicador mostra o número médio de anos que uma pessoa ao nascer em determinado município viveria a partir de seu nascimento, mantido os mesmos padrões de mortalidade. O seu cálculo é realizado pela fração entre a variação de expectativa de vida do município e a expectativa de vida ao nascer no País, através da seguinte forma, como por exemplo:

IDHM LONGEVIDADE (2) = (75 - 25) / (82 - 25). Onde 75 seria a expectativa máxima de vida ao nascer no município e 25 a expectativa mínima. Enquanto no denominador, 85 é a expectativa máxima de vida ao nascer no Brasil e 25 é a mínima.

O IDHM EDUCAÇÃO (2) que é obtido do índice de acesso ao conhecimento, é medido por meio de dois indicadores extraídos dos Censos Demográficos do IBGE:

- a escolaridade da população adulta - medida através do cálculo do percentual das pessoas de 18 anos ou mais de idade, com ensino fundamental completo e tem peso 1 ;

- fluxo escolar da população jovem - é medido pela média aritmética do percentual de crianças de 5 a 6 anos frequentando a escola, do percentual de jovens de 11 a 13 anos frequentando os anos finais do ensino fundamental, do percentual de jovens dos 15 aos 17 anos com ensino fundamental completo e do percentual de jovens dos 18 aos 20 anos com ensino médio completo. O resultado da média aritmética de todos esses percentuais tem peso 2 .

A média geométrica desses dois indicadores tem por resultado o IDHM EDUCAÇÃO (2). 
O IDHM RENDA (3), que é extraído do índice de padrão de vida, é medido pela renda municipal per capita, que é a renda média dos residentes de determinado município. É obtido através da soma da renda de todos os residentes do município, dividida pelo número de pessoas residentes naquele município, inclusive crianças e pessoas sem renda. Os dados também são extraídos dos Censos Demográficos do IBGE.

Dessa forma, quanto mais próximo do número um for o resultado da média geométrica dos três índices, IDHM LONGEVIDADE (1), IDHM EDUCAÇÃO (2) e IDHM RENDA (3), melhor é o Índice de Desenvolvimento Humano do Município - IDHM (Chediek et al. 2013).

No sentido de avaliar a aplicabilidade deste método no Brasil, pode-se agora trazer os dados mais recentes sobre o Índice de Desenvolvimento Humano - IDHM, começando pela visão territorial dos Estados Brasileiros.

Quadro 2 - Índice de Desenvolvimento Humano - IDHM dos Estados Brasileiros.

\begin{tabular}{|l|c|}
\hline \multicolumn{1}{|c|}{ Territorialidades } & IDHM 2017 \\
\hline Brasil & 0,778 \\
\hline Acre & 0,719 \\
\hline Alagoas & 0,683 \\
\hline Amapá & 0,74 \\
\hline Amazonas & 0,733 \\
\hline Bahia & 0,714 \\
\hline Ceará & 0,735 \\
\hline Distrito Federal & 0,85 \\
\hline Espírito Santo & 0,772 \\
\hline Goiás & 0,769 \\
\hline Maranhão & 0,687 \\
\hline Mato Grosso do Sul & 0,766 \\
\hline Mato Grosso & 0,774 \\
\hline Minas Gerais & 0,787 \\
\hline Paraíba & 0,722 \\
\hline Paraná & 0,792 \\
\hline Pará & 0,698 \\
\hline Pernambuco & 0,727 \\
\hline Piauí & 0,697 \\
\hline Rio de Janeiro & 0,796 \\
\hline Rio Grande do Norte & 0,731 \\
\hline Rio Grande do Sul & 0,787 \\
\hline Rondônia & 0,725 \\
\hline Roraima & 0,752 \\
\hline Santa Catarina & 0,808 \\
\hline São Paulo & 0,826 \\
\hline Sergipe & 0,702 \\
\hline Tocantins & 0,743 \\
\hline & \\
\hline & \\
\hline
\end{tabular}

Fonte: Atlas do Desenvolvimento Humano no Brasil (2020).

Dessa forma, pode-se perceber que Pernambuco ocupa a $17^{\mathrm{a}}$ posição no ranking nacional quanto ao IDHM, cuja classificação é de pontuação igual a 0,727 e foi feita com base no IDHM dos municípios que compõem cada Estado Brasileiro. Por sua vez, Recife ocupa a 53 ${ }^{\mathrm{a}}$ posição perante todos os municípios Pernambucanos, com o IDHM de 0,772, conforme demonstrado a seguir. 
Quadro 3 - Municípios do Estado de Pernambuco.

\begin{tabular}{|l|c|c|c|c|c|c|c|c|}
\hline Territorialidade & $\begin{array}{c}\text { Posição } \\
\text { IDHM }\end{array}$ & IDHM & $\begin{array}{c}\text { Posição } \\
\text { IDHM } \\
\text { Renda }\end{array}$ & $\begin{array}{c}\text { IDHM } \\
\text { Renda }\end{array}$ & $\begin{array}{c}\text { Posição } \\
\text { IDHM } \\
\text { Educação }\end{array}$ & $\begin{array}{c}\text { IDHM } \\
\text { Educação }\end{array}$ & $\begin{array}{c}\text { Posição } \\
\text { IDHM } \\
\text { Longevidade }\end{array}$ & $\begin{array}{c}\text { IDHM } \\
\text { Longevidade }\end{array}$ \\
\hline $\begin{array}{l}\text { Fernando de } \\
\text { Noronha (PE) }\end{array}$ & 37 & 0,788 & 56 & 0,839 & 31 & 0,748 & 57 & 0,781 \\
\hline Recife (PE) & 53 & 0,772 & 70 & 0,825 & 79 & 0,698 & 40 & 0,798 \\
\hline Olinda (PE) & 90 & 0,735 & 59 & 0,836 & 102 & 0,675 & 134 & 0,704 \\
\hline Paulista (PE) & 93 & 0,732 & 65 & 0,83 & 74 & 0,703 & 165 & 0,673 \\
\hline $\begin{array}{l}\text { Jaboatão dos } \\
\text { Guararapes (PE) }\end{array}$ & 108 & 0,717 & 65 & 0,83 & 135 & 0,642 & 146 & 0,692 \\
\hline Petrolina (PE) & 128 & 0,697 & 96 & 0,799 & 166 & 0,611 & 143 & 0,695 \\
\hline $\begin{array}{l}\text { Camaragibe } \\
\text { (PE) }\end{array}$ & 133 & 0,692 & 90 & 0,805 & 149 & 0,628 & 182 & 0,656 \\
\hline $\begin{array}{l}\text { Cabo de Santo } \\
\text { Agostinho (PE) }\end{array}$ & 139 & 0,686 & 83 & 0,812 & 168 & 0,609 & 184 & 0,654 \\
\hline Carpina (PE) & 145 & 0,68 & 89 & 0,806 & 158 & 0,619 & 208 & 0,63 \\
\hline $\begin{array}{l}\text { Abreu E Lima } \\
\text { (PE) }\end{array}$ & 146 & 0,679 & 104 & 0,791 & 145 & 0,632 & 213 & 0,625 \\
\hline
\end{tabular}

Fonte: Atlas do Desenvolvimento Humano no Brasil (2020).

O IDHM LONGEVIDADE foi o que trouxe a melhor posição para o Recife no ranking nacional e na análise do IDHM exclusivamente entre os municípios de Pernambuco, Recife fica na segunda posição, ficando atrás apenas de Fernando de Noronha devido às suas características diferenciadas e específicas.

É importante ressaltar que o Programa das Nações Unidas para o Desenvolvimento - PNUD, incentiva a adaptabilidade do IDHM, encorajando os Países a desenharem os IDHs nacionais que mais sejam adequados às suas necessidades (Chediek et al. 2013).

Por isso é possível que características e variáveis mais específicas quanto à definição de Cidades Inteligentes, além das que já compõem a equação geométrica do IDHM, possam vir a ser acrescentadas, direcionando ainda mais esse indicador em direção à análise proposta na pesquisa feita neste artigo.

A análise comparativa das conjunturas municipais e da sua evolução ao longo dos anos por meio de indicadores selecionados e comuns a todos, permite que pesquisadores e governantes avaliem ações, projetos e programas exitosos, bem como, redirecionem as políticas públicas em direção a melhores resultados (Chediek et al. 2013).

O site Atlas Brasil, no seu endereço - atlasbrasil.org.br/ranking, disponibiliza a ferramenta aberta em que é possível se elaborar a pesquisa sobre qualquer cidade, estado, região e comparar os dados sob a mesma base de dados escolhida e para qualquer um dos indicadores que compõem o IDHM. (atlasbrasil.org.br/ranking, 05.12.2020).

Através deste site foi possível verificar a seguinte classificação para as nove capitais do Nordeste: 
Quadro 4 - classificação para as nove capitais do Nordeste.

\begin{tabular}{|l|c|c|c|}
\hline \multicolumn{1}{|c|}{ Local } & Classificação NE & IDHM & Classificação BR \\
\hline Brasil & & 0,778 & $53^{\mathrm{a}}$ \\
\hline Recife & $1^{\mathrm{a}}$ & 0,772 & $55^{\mathrm{a}}$ \\
\hline Aracajú & $2^{\mathrm{a}}$ & 0,770 & $57^{\mathrm{a}}$ \\
\hline São Luiz & $3^{\mathrm{a}}$ & 0,768 & $62^{\mathrm{a}}$ \\
\hline Natal & $4^{\mathrm{a}}$ & 0,763 & $62^{\mathrm{a}}$ \\
\hline João Pessoa & $5^{\mathrm{a}}$ & 0,763 & $66^{\mathrm{a}}$ \\
\hline Salvador & $6^{\mathrm{a}}$ & 0,759 & $71^{\mathrm{a}}$ \\
\hline Fortaleza & $7^{\mathrm{a}}$ & 0,754 & $74^{\mathrm{a}}$ \\
\hline Teresina & $8^{\mathrm{a}}$ & 0,751 & $104^{\mathrm{a}}$ \\
\hline Maceió & $9^{\mathrm{a}}$ & 0,721 & ${ }^{2}$ \\
\hline
\end{tabular}

Fonte: atlasbrasil.org.br/ranking (2020).

Conforme citado anteriormente neste artigo, o Estudo da Urban Systems realizado em 2020, para fazer o ranking nacional das cidades inteligentes, aponta o Recife como a primeira entre as capitais do Nordeste do Brasil. Havendo nesse sentido a mesma classificação também encontrada pela análise do IDHM acima citada.

Para melhor caracterizar alguns fatores tecnológicos que são suporte à cidade do Recife nos aspectos de seu desenvolvimento, realizamos uma amostragem de assuntos que são aplicados na gestão da Prefeitura deste município, através de entrevista com um dos principais executivos da gestão da informação ao longo dos últimos anos até 2020, conforme relatado a seguir.

A empresa municipal de informática - EMPREL, é uma empresa pública com personalidade jurídica de direito privado, que faz parte da administração indireta da Prefeitura do Município do Recife e presta serviços de tecnologia da informação e de comunicação a todas as esferas da Prefeitura, contribuindo no planejamento estratégico do município. Desde 2013 vem fortalecendo a atuação direcionada ao cidadão, a partir de projetos como o portal de dados abertos, o portal da transparência, dentre outros que compõem a iniciativa do e-Gov e o conecta Recife, que fornece internet gratuita em diversas partes da cidade (Antunes, 2020).

Além dos esforços direcionados para a busca de soluções tecnológicas no âmbito da gestão pública e dentro do território nacional, a Prefeitura realiza investimentos na busca por parcerias internacionais. Dessa forma, iniciou uma parceria com um governo de um dos países da Europa ${ }^{1}$, com o objetivo de constituir um Hub, onde possa ser disponibilizado as diversas visões dos dados já catalogados na gestão do município, alcançando inclusive dados extragovernamentais. Isso possibilitará o acesso aos dados nas formas gráficas, em tabelas, dados estatísticos, permitindo o engajamento para pesquisas e estudos. O projeto está na fase de planejamento com previsão de lançamento em 2021 (Antunes, 2020).

São diversos os dados gerados na gestão municipal, por diversas e distintas áreas, que atualmente já podem ser acessados como dados abertos em vários dos seus portais, na forma mais granular possível para quem estiver acessando o banco de dados (Antunes, 2020). A parceria citada, caminha na direção de dotar de centralidade a gestão dos dados, com foco em três pontos fundamentais: Governança de dados - integração e centralização; Hub para a cidade - tendo a Prefeitura como âncora do processo; Ações de engajamento - para disponibilizar informações para a sociedade.

As principais bases de acesso para as informações e plataformas de serviços, que além de darem celeridade na gestão pública, cumprem a legislação nos termos da comunicação e transparência necessária à administração pública e servem de base de informação para as melhorias da própria gestão, estão disponibilizadas no Quadro 5.

\footnotetext{
${ }^{1}$ No contrato com o País da Europa, consta cláusula de confidencialidade, então não podemos falar mais do que ele autorizou (Antunes, 2020).
} 
Quadro 5 - bases de acesso para às informações e plataformas de serviços públicos.

\begin{tabular}{|l|l|}
\hline \multicolumn{1}{|c|}{ Nome da base } & \multicolumn{1}{c|}{ Site } \\
\hline Portal da Transparência - PCR & http://transparencia.recife.pe.gov.br \\
\hline Dados Recife - Portal de Dados Abertos & http://dados.recife.pe.gov.br \\
\hline Hacker Cidadão 8.0 & http://hackercidadao.rec.br/ \\
\hline Portal de Licenciamento Urbanístico & https:///icenciamento.recife.pe.gov.br/ \\
\hline Bate Pronto & https://batepronto.recife.pe.gov.br/ \\
\hline CTTU - Autarquia de Trânsito e Transporte Urbano & https://cttu.recife.pe.gov.br/zona-azul \\
\hline Serviços para o Cidadão & http://www2.recife.pe.gov.br/servico/app-conecta-recife \\
\hline Atende em casa COVID 19 & https://www.atendeemcasa.pe.gov.br/login \\
\hline Quem deve testar para - COVID 19 & https://testecovid19.recife.pe.gov.br/ \\
\hline Porto Digital D.A.D.O & https://dado.recife.br/ \\
\hline
\end{tabular}

Fonte: Autores (2020).

Pode-se destacar aqueles cujas características se enquadram nas definições de transformação digital, como os que envolvem os processos de licenciamento. No passado, o interessado precisava lidar com a burocracia pública em diversas secretarias, até obter a licença de funcionamento. Agora, todo o processo é feito na forma digital, sem que o cidadão precise se deslocar fisicamente para diversas áreas da Prefeitura e o processo tramita virtualmente nas diversas áreas relacionadas ao assunto específico de cada solicitação de funcionamento de atividades produtivas, industriais, comerciais ou de serviços, envolvendo as áreas de urbanismo, ambiente e saúde/sanitária. (Prefeitura do Recife, 2020a).

Nessa mesma linha, o portal denominado "Bate Pronto", ligado à secretaria de trânsito-companhia de trânsito e transporte urbano, permite que todo o procedimento de registro de ocorrência de acidente de trânsito, sem vítimas fatais, seja feito virtualmente (Bate Pronto, 2020). Da mesma forma a "Zona Azul" eletrônica, permitiu a melhor gestão das vagas de estacionamento público, eliminando os riscos de interferência de terceiros na disponibilização das vagas de estacionamento, dotando de comodidade, celeridade, segurança e transparência a gestão desse item importante na mobilidade urbana (Prefeitura do Recife, 2020b).

O "Conecta Recife" é outro exemplo de disponibilização da tecnologia para facilitar a vida do cidadão nas grandes cidades, oferecendo informações importantes para a comunidade local e para turistas, sob as mais diversas áreas de interesse da população (Prefeitura do Recife, 2020c).

A Secretaria de Meio Ambiente, desenvolveu um projeto pioneiro no Brasil, para aferir os níveis de emissão de gases de efeito estufa na cidade do Recife. O projeto foi desenvolvido em uma parceria com o ICLEI - Governos Locais pela Sustentabilidade, com a participação no projeto da ONU - habitat, que é financiado pela comissão europeia, com o objetivo de fomentar políticas de desenvolvimento de baixo carbono nas cidades, notadamente na África do Sul, Indonésia, Índia e Brasil. Através desse projeto, a Prefeitura do Recife teve seu primeiro inventário sobre emissão de gases do efeito estufa, podendo trabalhar, a partir de então, sobre os resultados apontados por aquele trabalho, onde foi possível identificar fatores importantes como o que indicou que em 2012 a cidade emitiu 3.120.425,74 tCO2, distribuídos pelas seguintes fontes de emissão (Prefeitura do Recife, 2020d).

De acordo com o $1^{\circ}$ Inventário de Emissões de Gases de Efeitos Estufa da cidade de Recife (Prefeitura do Recife, 2020e):

- Transporte $-65,2 \%$;

- Resíduos-19,4\%;

- Energia residencial-6,3\%;

- Energia comercial/institucional-3,8\%;

- Governo-0,3\%. 
Com base nessas e em outras informações geradas pelo estudo, foi possível desenvolver o Plano de Redução de Emissões de Gases de Efeito Estufa, com metas de curto, médio e longo prazo (Prefeitura do Recife, 2020f).

As plataformas e sites direcionados para as áreas de saúde e de educação, permitem o georreferenciamento de informações de forma contínua, aperfeiçoando as decisões e direcionando estratégias mais precisas para a implementação das políticas públicas. Nesse sentido, durante o período de crise provocada pela pandemia, foi possível na prática, verificar ações inovadoras no enfrentamento da crise, como o programa "Atende em Casa", no qual equipe médica faz de forma telemática uma análise prévia dos casos acometidos pelo covid-19, bem como a plataforma para agendamento de exames de testagem para covid19, tudo de forma direta e online (Pernambuco, 2020; Prefeitura do Recife, 2020g).

\section{Considerações Finais}

Diante do exposto, chegou-se a certas conclusões. No que se diz respeito ao conceito de Smart Cities, existem inúmeras expressões encontradas na literatura para definir o que é uma Smart City, o que além de criar lacunas que dificultam o entendimento também dificultam o amplo alcance referente aos efeitos de uma Smart City e, consequentemente, atrapalham na caracterização específica das Cidades Inteligentes.

Nesse sentido, não seria coerente restringir o conceito considerando apenas as questões locais, mesmo sabendo que em cada cidade pode existir problemas específicos, faz-se necessário desenvolver o conceito pensando também de forma global. Logo, Smart City é conceito em construção, o que se justifica, principalmente, em decorrência de se tratar de um fenômeno recente e se utiliza de tecnologias, que estão em constante evolução, fazendo com que o seu uso seja cada vez mais abrangente, em termos de tornar os serviços da cidade mais eficientes.

Da mesma forma que existem vários conceitos de Smart Cities, também é possível identificar na literatura especializada, uma diversidade de indicadores utilizados para a classificação daquelas cidades consideradas inteligentes. No entanto, alguns indicadores reúnem pontos em comuns, quais sejam: indicadores de sustentabilidade, sociais, econômicos, ambientais e de governança que devem ser considerados. Por exemplo, o Ranking Connected Smart Cities é composto por 70 indicadores em 11 eixos temáticos: Mobilidade, urbanismo, Meio Ambiente, Tecnologia e Inovação, Empreendedorismo, Educação, Saúde, Segurança, Energia, Governança e Economia (CBN RECIFE, 2020).

De acordo com o referido ranking, a cidade de Recife ficou na $8^{\mathrm{a}}$ posição no que se refere às áreas de Empreendedorismo. Quanto às áreas de Mobilidade e Acessibilidade, a capital alcançou a $9^{\circ}$ posição. No que diz respeito às áreas de Tecnologia e Inovação a cidade está em $11^{\circ}$ lugar. Outra boa colocação é acerca da área de Saúde, Recife ficou $12^{\mathrm{a}}$ posição em todo o país (CBN Recife, 2020).

O ranqueamento em questão coloca Recife como a cidade mais inteligente e conectada do Norte e Nordeste do Brasil, e isso se deve aos projetos, trabalhos e ações, apenas para exemplificar, conforme os acima citados, que colocam a capital no roteiro das Cidades Inteligentes no Brasil, justamente por contemplar ações de mobilidade urbana, sustentabilidade, inclusão social e digital, desenvolvimento sustentável e serviços direcionados à população, inclusive para uso de dados abertos e com perspectiva de atender às boas práticas de governança e transformação digital.

Dessa forma, a contribuição aqui trazida implica em ter por referência um indicador que seja abrangente nacionalmente para permitir a comparabilidade dos dados, que possa ser usado para avaliar as políticas de desenvolvimento da região e das cidades, que possa ser desagregado e adaptado para avaliar características específicas que se pretenda dar maior atenção e que sirva de conceito comum para ser aplicado por todos.

Ao se detalhar alguns dos indicadores aplicados nos estudos aqui apresentados e a perspectiva de fazer uso de ferramentas estatísticas, demográficas, sociais, econômicas, de educação, saúde, mobilidade, sustentabilidade e de qualidade de vida, com o objetivo de melhor definir o conceito e a classificação de Cidades Inteligentes, pode-se esperar que estudiosos, 
pesquisadores, gestores e investidores tenham à sua disposição instrumentos capazes de contribuir significativamente para o processo de tomada de decisão sobre temas relacionadas às Cidades Inteligentes e ao Desenvolvimento de uma maneira geral.

Como este trabalho não teve um escopo mais abrangente sobre cada um dos indicadores possíveis de maior detalhamento, cabe a sua complementação em itens específicos que possam dar uma margem maior de avaliação sobre o tema das Smart Cities. Para aperfeiçoar o estudo ora apresentado, é possível aprofundar a dimensão da aplicabilidade de indicadores sob várias outras óticas e abordagens que certamente trarão maior dimensão para os resultados aqui delineados, como também é possível elaborar consulta formal aos elaboradores do Programa das Nações Unidas para o Desenvolvimento - PNUD, no sentido de sugerir adaptações que contemplem outros indicadores dirigidos à análise das Cidades Inteligentes.

Como desdobramentos da pesquisa percebe-se a possibilidade do aprofundamento de aspectos relacionados a movimentação nas cidades inteligentes, seja na perspectiva da movimentação de produtos e mercadorias, questões relacionadas a última milha, bem como, no que diz respeito a mobilidade das pessoas. Estes são pontos fundamentais para a agregação de bem-estar na cidade do Recife, a partir da agregação de inteligência trazida pelo conceito de cidades inteligentes.

\section{Referências}

Antunes, E. J. B. (2020). Entrevista. Entrevistador: Elcio Ricardo Leite Guimarães.

Atlas do Desenvolvimento Humano no Brasil. (2020). Pnud Brasil, Ipea e FJP, 2020. http://atlasbrasil.org.br/acervo/biblioteca.

Bachendorf, C. F. (2018). Inteligência, sustentabilidade e inovação nas cidades: uma análise da mobilidade urbana de Pato Branco - PR. 2018.152 f. Dissertação (Mestrado em Desenvolvimento Regional) - Universidade Tecnológica Federal do Paraná, Pato Branco.

Bate Pronto. (2020). Como funciona? https://batepronto.recife.pe.gov.br

Caragliu, A., Del Bo, C. \& Nijkamp, P. (2011). Smart Cities in Europe. Journal of Urban Technology, 18(2), 65-82. http://dx.doi.org/10.1080/10630732.2011.601117

CBN Recife. (2020). Recife é apontada como a cidade mais inteligente e conectada do Norte e Nordeste. https://www.cbnrecife.com/movimentoeconomico/artigo/recife-e-apontada-como-a-cidade-mais-inteligente-e-conectada-do-norte-e-nordeste

Chediek, J. et al. (2013). Índice de Desenvolvimento Humano Municipal Brasileiro. Brasília: PNUD: Ipea: FJP. http://www.atlasbrasil.org.br/2013/data/rawData/publicacao_atlas_municipal_pt.pdf.

Dameri, R. P. (2017). Smart City Implementation. Ebook: Springer.

Damiani, C., Machado, G. M. \& Gasparini, I. (2019). Personalização e Gamificação no Contexto de Cidades Inteligentes. Cadernos de Informática, 10(1).

Geertman, S., Allan, A., Pettit, C. \& Stillwell, J. (2017). Introduction to 'Planning Support Science for Smarter Urban Futures'. Springer.

Giffinger, R. \& Haindlmaier, G. (2010). Smart cities ranking: an effective instrument for the positioning of cities? ACE: Architecture, City and Environment. 4. https://www.researchgate.net/publication/228915976_

Gil, A. C. (2007). Como elaborar projetos de pesquisa. (4a ed.), Atlas.

IBGE, Instituto Brasileiro de Geografia e Estatística. (2010). Brasil/Pernambuco/Recife. https://cidades.ibge.gov.br/brasil/pe/recife/panorama

IBGE, Instituto Brasileiro de Geografia e Estatística. (2018). Brasil/Pernambuco/Recife/Frota de veículos. https://cidades.ibge.gov.br/brasil/pe/recife/pesquisa/22/28120

IBGE, Instituto Brasileiro de Geografia e Estatística. (2015). Pesquisa Nacional por Amostra de Domicílios - PNAD. https://educa.ibge.gov.br/jovens/conhecao-brasil/populacao/18313-populacao-rural-e-urbana.html

IBGE, Instituto Brasileiro de Geografia e Estatística. (2020). População. 2020. https://www.ibge.gov.br/apps/populacao/projecao/

Hall, R., Bowerman, B., Braverman, J., Taylor, J., Todosow, H. \& Wimmersperg, U. (2000). The vision of a smart city. In Proceedings of the 2nd International Life Extension Technology Workshop (p. 1-6). https://www.researchgate.net/publication/241977644_The_vision_of_a_smart_city

Harrison, C. \& Donnelly, I. (2011). A Theory of Smart Cities. In 55th Annual Meeting of the International Society for the Systems Sciences 2011. http://journals.isss.org/index.php/proceedings55th/article/ viewFile/1703/572

Harvey, D. (1992). Condição pós-moderna: Uma Pesquisa sobre as Origens da Mudança Cultural. Edições Loyola, (25a ed.).

Jornal Oficial da União Europeia. (2020). Parecer do Comité das Regiões Europeu Cidades inteligentes: novos desafios para uma transição justa rumo à neutralidade climática — Como aplicar os ODS na prática? C 39/70, 5.2.2020. https://eur-lex.europa.eu/legalcontent/PT/TXT/PDF/?uri=CELEX:52019IR2974\&from=EN 
Kitchin, R., Lauriault, T. P. \& Mcardle, G. (2015). Knowing and governing cities through urban indicators, city benchmarking and real-time dashboards. Regional Studies, Regional Science, 2(1), 6-28.

Lemos, A. (2013). Cidades inteligentes. Caderno especial: Espaços urbanos, 12(2). http://bibliotecadigital.fgv.br/ojs/index.ph p/gvexecutivo/article/view/20720/19454

Maclaren, V. W. (1996). Urban Sustainability Reporting. Journal of the American Planning Association, 62(2), 184-202.

Pereira, A. S., Shitsuka, D. M., Parreira, \& F. J. Shitsuka, R. (2018). Metodologia da pesquisa científica. [e-book]. Santa Maria: UAB/NTE/UFSM. https://www.ufsm.br/app/uploads/sites/358/2019/02/Metodologia-da-Pesquisa-Cientifica_final.pdf

Pernambuco. (2020). Atende em casa. https://www.atendeemcasa.pe.gov.br/login

Prefeitura do Recife. (2020e). $1^{\circ}$ Inventário de Emissões de Gases de Efeitos Estufa da cidade de Recife. http://www2.recife.pe.gov.br/sites/default/files/4._primeiro_inventario_de_emissao_de_gases_de_efeito_estufa_da_cidade_do_recife.pdf

Prefeitura do Recife. (2020c). App conecta Recife. http://www2.recife.pe.gov.br/servico/app-conecta-recife

Prefeitura do Recife. (2020d). Mudanças climáticas. http://www2.recife.pe.gov.br/taxonomy/term/9099?op=NTI5Mg==

Prefeitura do Recife. (2020f). O caminho certo para Recife Brasil Implementando diretrizes espaciais de apoio ao desenvolvimento urbano de baixa emissão. http://www2.recife.pe.gov.br/sites/default/files/diretrizes_de_baixo_carbono_para_plano_diretor.pdf

Prefeitura do Recife. (2020a). Portal de licenciamento urbanístico. https://licenciamento.recife.pe.gov.br

Prefeitura do Recife. (2020g). Quem deve testar para COVID - 19? https://testecovid19.recife.pe.gov.br

Prefeitura do Recife. (2020b). Zona azul. https://cttu.recife.pe.gov.br/zona-azul

REDE social de cidades. https://www.redesocialdecidades.org.br/br/PE/recife/congestionamento

Rizzon, F., Bertelli, J., Matte, J., Graebin, R. E. \& Macke, J. (2017). Smart City: um conceito em construção. Revista Metropolitana de Sustentabilidade - RMS, São Paulo, 7(3), p. 123-42.

Rossi, M. (2018). Recife, o pior trânsito “em linha reta” do Brasil. El País. https://brasil.elpais.com/brasil/2018/06/26/politica/1530040918_051796.html

Rother, E. T. Editorial. (2007). Acta Paulista de enfermagem, 20(2).

Santos, Mi. (2000). Por uma outra globalização. Record, (30a ed.).

Silva, C. C. et al. (2020). Acesso e utilização de serviços odontológicos por gestantes: revisão integrativa de literatura. Ciênc. saúde coletiva, Rio de Janeiro, 25(3), p. 827-35. http://www.scielo.br/scielo.php?script=sci_arttext\&pid=S1413-81232020000300827\&lng=en\&nrm=iso

Slomski, V. (2003). Governança e Custos no Setos Público Governamental; Contabilidade Pública: Teoria e Prática. Atlas.

Tokmakoff, A. \& Billington, J. (1999). Consumer Services in Smart City Adelaide. In K. Bjerg\& K. Borreby (Eds.), Paper published at HOIT 94. Proceedings of an International Cross-disciplinary Conference on Home-Oriented Informatics, Telematics \& Automation, University of Copenhagen. https://www.researchgate.net/publication/2466715_Consumer_Services_in_Smart_City_Adelaide/citation/download

Toledo, M. (2017). No horário de pico, trânsito do Recife é o mais lento do Brasil. Folha de Pernambuco, 2017. https://www.folhape.com.br/noticias/no-horariode-pico-transito-do-recife-e-o-mais-lento-do-brasil/51118/

Torres, H. G. (2016). A política habitacional e a metrópole: exclusão e inclusão dos mais fracos? $1^{a}$ Analise Seade.

Washburn, D., Sindhu, U., Balaouras, S., Dines, R. A., Hayes, N. M. \& Nelson, L. E. (2020). Helping CIOs Understand "Smart City” Initiatives: Defining the Smart City, Its Drivers, and the Role of the CIO. Cambridge, MA: Forrester Research. http://public.dhe.ibm.com/partnerworld/pub/ smb/smarterplanet/forr_help_cios_und_smart_city_initiatives.pdf 